\title{
Anti-inflammatory properties of betulinic acid and xylopic acid in the carrageenan-induced pleurisy model of lung inflammation in mice
}

\author{
Edmund Ekuadzi ${ }^{1}$, Robert P Biney ${ }^{2}$, Charles K Benneh ${ }^{3}$, Bismark Osei Amankwaa ${ }^{1}$, Jonathan Jato ${ }^{4}$ \\ ${ }^{\text {I} P h a r m a c o g n o s y, ~ K w a m e ~ N k r u m a h ~ U n i v e r s i t y ~ o f ~ S c i e n c e ~ a n d ~ T e c h n o l o g y, ~ G h a n a, ~}{ }^{2}$ Department of Pharmacology, \\ School of Medical Sciences, University of Cape Coast, Cape Coast, Ghana, ${ }^{3}$ Department of Pharmacology, School of \\ Pharmacy, University of Health and Allied Sciences, Ho, Ghana, ${ }^{4}$ Department of Pharmacognosy, School of Pharmacy, \\ University of Health and Allied Sciences, Ho, Ghana
}

Margaritaria discoidea and Xylopia aethiopica are plants native in the Ghana and the West-African region and used traditionally to treat different pathologies including inflammatory conditions. Betulinic acid (BA) and xylopic acid (XA) extracted from M. discoidea and X. aethiopica respectively have been shown to possess anti-inflammatory effects. However, their specific effect on lung inflammation and their ability to modulate oxidative challenge under such circumstances is yet to be reported. Consequently, we examined the effect of the two plant-derived acids in lung inflammation with the carrageenan-induced pleurisy model and further elucidate selected downstream mechanism(s) of this anti-inflammatory effect with specific focus on pleural exudate formation, in vivo oxidative stress markers and histopathological changes in lung tissue.

The anti-inflammatory effect of BA and XA were established by an in vivo assay using the carrageenan-induced pleural inflammation model in mice. Also, the ability of BA and XA to increase catalase, superoxide dismutase, glutathione levels and decrease lipid peroxidation level in reactive oxidative assays were assessed. In addition, the ability of XA and BA to prevent potential lung tissue damage was quantified.

Pre-treatment with BA and XA reduced significantly, signs of inflammation: neutrophil infiltration, oedema and alveoli septal thickening in carrageenan-treated lung tissue. Additionally, BA or XA pre-treatment lowered the degree of lipid peroxidation in the lung tissue while increasing the levels of catalase, superoxide dismutase and glutathione in vivo. Comparatively, XA was more efficacious than BA in the prevention of lung tissue damage.

$\mathrm{XA}$ and BA derived from Xylopia aethiopica and Margaritaria discoidea possess anti-inflammatory and in vivo antioxidant activities in mice pleurisy model. The effect of these compounds gives credence to the traditional use in the management of inflammatory conditions of the airway. 